\title{
A Robust Multigrid Method for a Discretization of the Incompressible Navier-Stokes Equations in General Coordinates
}

\author{
C. W. Oosterlee AND P. Wesseling \\ Delfi University of Technology, P.O. Box 5031, 2600 GA Delft. The Netherlands
}

Received March 2, 1992

C. W. Oosterlee and P. Wesseling, A Robust Multigrid Method for Discretization of the Incompressible Navier-Stokes Equations in General Coordinates, IMPACT of Commuting in Science and Engineering, 5, 128-151 (1993).

The stationary incompressible Navier-Stokes equations are discretized with a finite volume method in curvilinear coordinates. The arbitrarily shaped domain is mapped onto a rectangular block, resulting in a boundary-fitted grid. In order to obtain accurate discretizations of the transformed cquations certain requirements on geometric quantities should be met. The choice of velocity components is of importance. Contravariant flux unknowns and the pressure are used as primary unknowns on a staggered grid. The system of discretized equations is solved with a nonlinear multigrid algorithm, in which a robust line smoother, called Symmetric Coupled Alternating Lines, is implemented. All unknowns on a line of cells are updated simultaneously with alternating zebra sweeping. The solution algorithm shows satisfying average reduction factors for arbitrary domains, cven when highly stretched cells are present. (c) 1993 Academic Press. Inc.

\section{INTRODUCTION}

One way to approximate flows in complicated geometries is to discretize the incompressible Navier-Stokes equations in general coordinates. Using a finite volume or a finite difference discretization method, it is not at all trivial to obtain accurate discretizations in boundary-fitted coordinates for arbitrarily shaped domains. Several choices have to be made, for example, between contravariant, covariant, Cartesian, or other velocity components, and between a staggered and nonstaggered grid arrangement. Recent articles in which one of these approaches is adopted are $[4,7,8,19,21]$. We choose a staggered grid with contravariant flux components as velocity unknowns. Discretization accuracy depends strongly on the way in which geometric quantities related

0899-8248/93\$5.00 


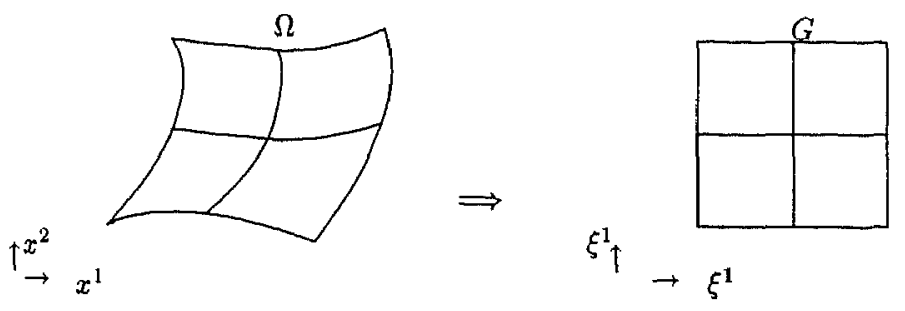

FIG. 2.1. The mapping of a physical domain $\Omega$ onto a rectangular block $G$.

to the coordinate mapping are discretized. It is assumed that the mapping is given only in terms of the physical coordinates associated with the points in the uniform computational grid. The geometric quantities require differentiation of the mapping once (for the base vectors and the metric tensor) or twice (for Christoffel symbols). The resulting work and the inaccuracy which occurs if certain rules are not followed have led many authors to consider noncoordinate-invariant discretizations and/or colocated (nonstaggered) grid arrangements. This usually compromises generality, stability and robustness. It seems attractive to use coordinate invariant discretizations of physical laws, provided discretization accuracy can be maintained, and this is the approach followed here. Discretization accuracy can be maintained if the geometric quantities are discretized in a special way, and if flux components rather than velocity components are used as velocity unknowns. In Section 2 the discretization and the requirements to obtain accuracy are presented.

Major developments have taken place in the domain of solution algorithms for discretized equations. For general problems, in principle the fastest solution method to solve the system is the nonlinear multigrid method. Many articles have appeared in which multigrid methods are presented for the NavierStokes equations in Cartesian coordinates [3, 5, 24, 26-28, 31, 32, 35]. Many methods are not robust, usually due to lack of robustness of the smoother. Difficulties arise at high Reynolds numbers or in problems in which stretched cells occur. However, due to numerical grid generation procedures and also for accuracy reasons highly stretched cells (which causes strong coupling of some unknowns) are not unusual in arbitrarily shaped domains, and therefore robustness of the smoothing method is a necessary requirement. A survey of some smoothing methods is given in Section 3, where the multigrid method and the smoothing method used are also presented. Some test problems and results are presented in Section 4 to show accurate solutions and good convergence rates.

\section{THE INCOMPRESSIBLE NAVIER-STOKES EQUATIONS IN GENERAL COORDINATES}

We use boundary-fitted coordinates, mapping the arbitrarily shaped domain $\Omega$ onto a rectangle $G$, as shown in Figure $2.1 ; \mathbf{x}$ are Cartesian coordinates, $\boldsymbol{\xi}$ 
boundary conforming curvilinear coordinates. In order to obtain a coordinateinvariant formulation tensor analysis is used $[1,25]$. We briefly recall a few facts that will be used in the sequel.

Covariant base vectors $\mathbf{a}_{(\alpha)}$, contravariant base vectors $\mathbf{a}^{(\alpha)}$, the covariant and contravariant metric tensors $g_{\alpha \beta}$ and $g^{\alpha \beta}$ are defined as

$$
\mathbf{a}_{(\alpha)}=\frac{\partial \mathbf{x}}{\partial \xi^{\alpha}}, \quad \mathbf{a}^{(\alpha)}=\frac{\partial \xi^{\alpha}}{\partial \mathbf{x}}, g_{\alpha \beta}=\mathbf{a}_{(\alpha)} \cdot \mathbf{a}_{(\beta)}, \quad g^{\alpha \beta}=\mathbf{a}^{(\alpha)} \cdot \mathbf{a}^{(\beta)}
$$

The determinant of the covariant metric tensor $g_{\alpha \beta}$ is denoted by $g ; \sqrt{g}$ equals the Jacobian of the transformation given by

$$
\sqrt{g}=a_{(1)}^{1} a_{(2)}^{2}-a_{(1)}^{2} a_{(2)}^{1}
$$

Summation over repeated Greek indices is implied.

The covariant derivative of a contravariant tensor of rank one is defined as

$$
Q_{, \beta}^{\alpha}=\frac{\partial Q^{\alpha}}{\partial \xi^{\beta}}+\{\underset{\gamma \beta}{\alpha}\} Q^{\gamma}
$$

where $\left\{{ }_{\gamma^{\beta}}^{\alpha}\right\}$ represents the Christoffel symbol of the second kind, defined by

$$
\left\{\begin{array}{l}
\alpha \gamma \\
\beta \gamma
\end{array}=\left\{{ }_{\gamma \beta}^{\alpha}\right\}=\mathbf{a}^{(\alpha)} \cdot \frac{\partial \mathbf{a}_{(\gamma)}}{\partial \xi^{\beta}}=\frac{\partial \xi^{\alpha}}{\partial x^{\delta}} \frac{\partial^{2} x^{\delta}}{\partial \xi^{\gamma} \partial \xi^{\beta}} .\right.
$$

It can be shown that

$$
Q_{, \alpha}^{\alpha}=\frac{1}{\sqrt{g}} \frac{\partial \sqrt{g} Q^{\alpha}}{\partial \xi^{\alpha}}
$$

Further it can be shown that

$$
\left(\sqrt{g} Q^{\alpha}\right)_{, \beta}=\frac{\partial \sqrt{g} Q^{\alpha}}{\partial \xi^{\beta}}+\left\{{ }_{\gamma \beta}^{\alpha}\right\} \sqrt{g} Q^{\gamma}-\left\{\gamma_{\gamma \beta}^{\gamma}\right\} \sqrt{g} Q^{\alpha} .
$$

The covariant derivative of a tensor of rank two is defined as

$$
Q_{, \gamma}^{\alpha \beta}=\frac{\partial Q^{\alpha \beta}}{\partial \xi^{\gamma}}+\left\{\begin{array}{c}
\alpha \delta \\
\gamma^{\delta}
\end{array}\right\} Q^{\delta \beta}+\left\{\begin{array}{c}
\beta \\
\gamma \delta
\end{array}\right\} Q^{\alpha \delta} .
$$


It can be shown that

$$
Q_{, \beta}^{\alpha \beta}=\frac{1}{\sqrt{g}} \frac{\partial \sqrt{g} Q^{\alpha \beta}}{\partial \xi^{\beta}}+\{\underset{\gamma \beta}{\alpha}\} Q^{\gamma \beta}
$$

In general curvilinear coordinates the stationary incompressible Navier-Stokes equations are given by

$$
\begin{gathered}
U_{, \alpha}^{\alpha}=0 \\
T_{, \beta}^{\alpha \beta} \equiv\left(\rho U^{\alpha} U^{\beta}\right)_{, \beta}+\left(g^{\alpha \beta} p\right)_{, \beta}-\tau_{, \beta}^{\alpha \beta}=\rho F^{\alpha},
\end{gathered}
$$

where $\rho$ is the fluid density and $\tau^{\alpha \beta}$ represents the deviatoric stress tensor given by

$$
\tau^{\alpha \beta \beta}=\mu\left(g^{\alpha \gamma} U_{, \gamma}^{\beta}+g^{\gamma \beta} U_{, \gamma}^{\alpha}\right)
$$

with $\mu$ the viscosity coefficient.

The convection tensor is linearized using a Picard iteration:

$$
\left(\rho U^{\alpha x} U^{\beta}\right)_{, \beta} \simeq\left(\rho U^{\alpha(n+1)} U^{\beta(n)}\right)_{, \beta} .
$$

The convection term is discretized with a so-called hybrid discretization scheme [14]. Depending on the mesh-Reynolds-number $\operatorname{Re}_{\alpha}^{(i, j)}$ (i.e., the ratio between the absolute magnitudes of the flux part of the convection term and the viscous term in point $(i, j)$ in the $\xi^{\text {r }}$-direction) the flux part of the convection term is discretized with a central difference scheme (when $\operatorname{Re}_{\alpha}^{(i, j)}<$ 1) or with a first-order upwind scheme (when $\operatorname{Re}_{\alpha}^{(i, j)}>1$ ).

In order to obtain accurate discretizations on nonsmooth grids, some requirements should be met $[11,13,22,33]$ :

(i) The geometric identity $\oint_{(;} a_{\beta}^{(\alpha)} d S_{(\alpha)}=0$ (coming from the application of the divergence theorem to a constant vector field) should be satisfied numerically. ( $d S_{(\alpha)}$ represents the physical surface element.) This requirement imposes rules on the numerical approximation of covariant and contravariant base vectors.

(ii) When representing a constant velocity field $\mathbf{u}$ on the staggered grid in terms of its contravariant components $U^{\alpha}$, and recomputing $\mathbf{u}$ from $U^{\alpha}$, the original vector field $\mathbf{u}$ should be recovered exactly.

(iii) Uniform flow fields should satisfy the discrete equations exactly. From this requirement the use of the contravariant flux components $V^{\alpha}=$ $\sqrt{g} \cdot U^{\alpha}$, a relative contravariant tensor of weight one, as velocity unknowns is found to be preferable, instead of $U^{\alpha}$, although this by itself is not sufficient 
to meet this requirement; we will not go further into this here. Details can be found in [22].

The first two requirements can be met if one proceeds as follows. The base vectors $\mathbf{a}_{(\alpha)}$ are computed according to

$$
a_{(1)}^{\beta}=\frac{\delta x^{\beta}}{\delta \xi^{1}}, \quad a_{(2)}^{\beta}=\frac{\delta x^{\beta}}{\delta \xi^{2}}
$$

in the $V^{2}$ - and $V^{1}$-points, respectively, in the staggered grid to be presented shortly. Furthermore,

$$
\mathbf{a}^{(1)}=\frac{1}{\sqrt{g}}\left(a_{(2)}^{2},-a_{(2)}^{1}\right), \quad \mathbf{a}^{(2)}=\frac{1}{\sqrt{g}}\left(-a_{(1)}^{2}, a_{(1)}^{1}\right),
$$

taking averages where required.

For convenience we introduce the local cell coordinates given by Figure 2.2 , which shows part of the computational grid in the $\xi$-plane. Integration of the incompressibility constraint over a pressure cell with center at $(0,0)$ gives, using (2.5) and $\partial \Omega=\sqrt{g} d \xi^{\prime} d \xi^{2}$ :

$$
\int_{\Omega} U_{, \alpha}^{\alpha} d \Omega=\int_{G} \frac{\partial \sqrt{g} U^{\alpha}}{\partial \xi^{\alpha}} d \xi^{1} d \xi^{2}
$$

Discretization of (2.15) gives

$$
\left.\int_{G} \frac{\partial \sqrt{g} U^{\alpha}}{\partial \xi^{\alpha}} d \xi^{1} d \xi^{2} \cong V^{1}\right|_{-1,0} ^{1,0} \delta \xi^{2}+\left.V^{2}\right|_{0,-1} ^{0,1} \delta \xi^{1},
$$

where $V^{\alpha}=\sqrt{g} U^{\alpha}$. Let $\mathbf{u}$ be a constant vector field. Substituting $V^{\alpha}=$ $\sqrt{g} a_{\beta}^{(\alpha)} u^{\beta}$ and using (2.14) one finds that

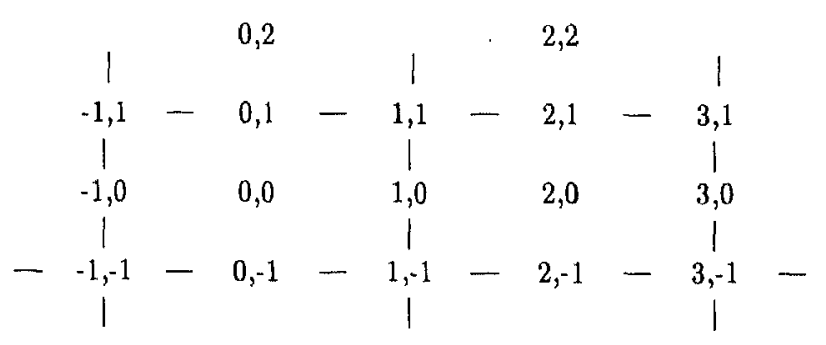

FIG. 2.2. Local cell coordinates. 


$$
\left.V^{1}\right|_{-1,0} ^{1,0} \delta \xi^{2}+\left.V^{2}\right|_{0 ;-1} ^{0,1} \delta \xi^{1}=0
$$

so that requirement (i) is satisfied. Requirement (ii) is verified as follows. Let $w$ be a constant vector field. Its representation in terms of $V^{\alpha}$ on the staggered grid is $V^{\alpha}=\sqrt{g} a_{\beta}^{(\alpha)} w^{\beta}$. Hence, using (2.14),

$$
V^{1}=a_{(2)}^{2} w^{1}-a_{(2)}^{1} w^{2}, \quad V^{2}=-a_{(1)}^{2} w^{1}+a_{(1)}^{1} w^{2} .
$$

Now recompute the Cartesian components $u^{\alpha}$ from (2.18) in the cell vertices,

$$
\left.u^{\alpha}\right|_{1,1}=\left.\frac{1}{2 \sqrt{g}}\right|_{1,1}\left\{\sum_{1}\left(a_{(1)}^{\alpha} V^{1}\right)+\sum_{2}\left(a_{(2)}^{\alpha} V^{2}\right)\right\},
$$

where $\Sigma_{1}$ indicates summation over grid points $(1,0)$ and $(1,2)$, and $\Sigma_{2}$ indicates summation over $(0,1)$ and $(2,1)$. Substitution of $(2.18)$ in $(2.19)$, and evaluation of $\sqrt{g}$ according to (2.2) results in

$$
\left.u^{\alpha}\right|_{1,1}=\left.w^{\alpha}\right|_{1,1} .
$$

We also have (2.20) in cell centers $(0,0)$. Hence, requirement (ii) is satisfied. If $U^{\alpha}$ is used as primary unknown instead of $V^{\alpha}(2.20)$ would not hold exactly, which is why the use of $V^{*}$ is to be preferred. This is confirmed by numerical experiments on the Navier-Stokes equations. With use of $V^{\text {ar }}$ the covariant differentiation rule of (2.6) should be used.

Requirement (iii) is not fully met in our discretization. The consequence is that inaccuracies may show up where the mesh-size changes rapidly, but on reasonably smooth grids the accuracy is satisfactory; results will be presented later.

As a preparation for the discretization of the momentum equations we discuss the discretization of a general conservation law of the form

$$
T_{, \beta}^{\alpha \beta^{3}}=f^{\alpha} .
$$

This equation is to be integrated over finite volumes. On the staggered grid used here, integration takes place over cells with vertices in $U^{2}$-points and center in a $U^{1}$-point for $\alpha=1$, and vice versa for $\alpha=2$. Taking a cell with center at $(1,0)$ as an example finite volume discretization gives, using $(2.8)$,

$$
\begin{aligned}
\int_{\Omega} T_{, \beta}^{\mid \beta} d \Omega= & \int_{\Omega} \frac{\partial \sqrt{g} T^{1 \beta}}{\partial \xi^{\beta}} d \xi^{1} d \xi^{2}+\int_{\Omega}\left\{\gamma_{\gamma \beta}\right\} T^{\gamma \beta} \sqrt{g} d \xi^{1} d \xi^{2} \\
\cong & \left.\left(\sqrt{g} T^{11}\right)\right|_{0,0} ^{2,0} \delta \xi^{2}+\left.\left(\sqrt{g} T^{12}\right)\right|_{1,-1} ^{1} \delta \xi^{1} \\
& +\left(\left.\sqrt{g}\left\{\begin{array}{l}
1 \\
\gamma \beta
\end{array}\right\} T^{\gamma \beta}\right|_{1,0}\right) \delta \xi^{1} \delta \xi^{2} .
\end{aligned}
$$

With $T_{\beta, \beta}^{\alpha \beta}$ from (2.10) this is the discretization used for the momentum equations. It is found that the variable $V^{\alpha}=\sqrt{g} U^{\alpha}$ appears naturally in many places in (2.22). 
The total number of variables linked together in a momentum equation is 19. Figure 2.3 shows the staggered grid and for the $V^{1}$-momentum equation the 19-point molecule with indices. The linearized system of discrete equations can be represented in vector notation by

$$
\left(\begin{array}{ccc}
A^{11} & A^{12} & A^{13} \\
A^{21} & A^{22} & A^{23} \\
A^{31} & A^{32} & 0
\end{array}\right)\left(\begin{array}{c}
\mathbf{V}^{1} \\
\mathbf{V}^{2} \\
\mathbf{p}
\end{array}\right)=\left(\begin{array}{c}
\mathbf{F}^{1} \\
\mathbf{F}^{2} \\
0
\end{array}\right) \quad \text { or } \quad A \mathbf{V}=\mathbf{F}
$$

In the general nonorthogonal case $A^{11}$ and $A^{22}$ contain nine nonzero elements per row, $A^{12}$ and $A^{21}$ four, $A^{13}$ and $A^{23}$ six and $A^{31}$ and $A^{32}$ contain two nonzero elements per matrix row. On orthogonal grids $A^{11}$ and $A^{22}$ reduce to five and $A^{13}$ and $A^{23}$ to two nonzero elements per row.

\section{The NONLinear Multigrid Algorithm}

The discretized equations are solved with the standard nonlinear multigrid algorithm $[2,6]$. Details of this algorithm are presented in $[13,32]$. Here a robust smoother to cope with stretched cells and arbitrary Reynolds numbers is presented. In the system of discretized equations (2.23) a zero block appears on the main diagonal. Therefore $A$ is not an $M$-matrix, and iterative methods based on a splitting $A=M-N$ according to

$$
M \mathbf{V}^{n+1}=N \mathbf{V}^{n}+\mathbf{F}
$$

will not converge in general.

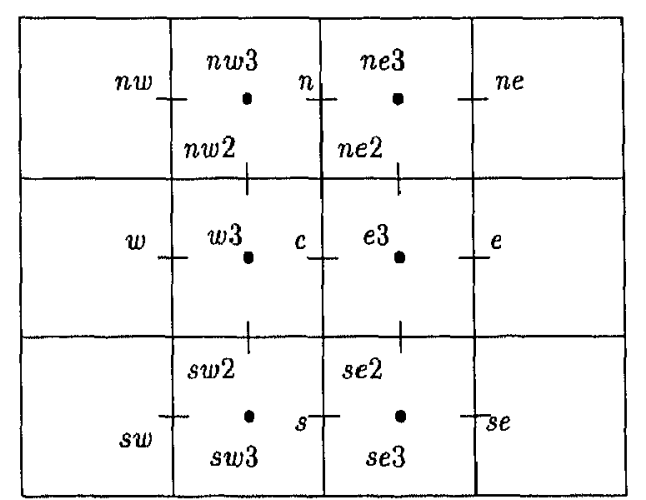

- $V^{1}$-flux unknown

| $V^{2}$-flux unknown

- -pressure unknown

Fig. 2.3. Staggered grid and molecule for the $V^{1}$ momentum equation. 
In order to solve these systems of equations iteratively matrix $A$ can be postconditioned by a matrix $B$, such that the zero block disappears; the situation is too complex to guarantee that $A B$ will be an $M$-matrix. The resulting system can now be split as follows:

$$
A B=M-N .
$$

This gives rise to the so-called distributed iteration methods

$$
M B^{-1} \mathbf{V}^{n+1}=N B^{-1} \mathbf{V}^{n}+\mathbf{F} .
$$

In the context of distributed iteration methods three methods have been used as smoothing methods in a multigrid algorithm $[34,35]$. With

$$
B=\left[\begin{array}{ccc}
I & 0 & -\left(S^{11}\right)^{-1} A^{13} \\
0 & I & -\left(S^{22}\right)^{-1} A^{23} \\
0 & 0 & I
\end{array}\right],
$$

where $\left(S^{i i}\right)^{-1}$ is an easy to evaluate approximation to $\left(A^{i i}\right)^{-1}$, we find the SIMPLE-type algorithms [15]. They are used as smoother in Cartesian coordinates in [24], and recently in arbitrarily shaped domains in [18]. Difficulties with SIMPLE are found when the Reynolds number is high, due to the diagonal term in matrix $A B:-\left(A^{31}\left(S^{11}\right)^{-1} A^{13}+A^{32}\left(S^{22}\right)^{-1} A^{23}\right)$, which approaches zero for $\mathrm{Re} \rightarrow \infty$ [35]. Therefore underrelaxation parameters are introduced depending on the shape of the domain and the Reynolds number, but robustness is impaired. With

$$
B=\left[\begin{array}{ccc}
I & 0 & A^{13} \\
0 & I & A^{23} \\
0 & 0 & A^{31} A^{13}+A^{32} A^{23}
\end{array}\right],
$$

the DGS (Distributive Gauss-Seidel) method [3] is obtained.

Difficulties arise because of the off-diagonal terms in $A B: A^{11} A^{13}+A^{12} A^{23}$ $+A^{13}\left(A^{31} A^{13}+A^{32} A^{23}\right)$ and $A^{21} A^{13}+A^{22} A^{23}+A^{23}\left(A^{31} A^{13}+A^{32} A^{23}\right)$, which can be dominant over the diagonal term, for example for certain values of the Reynolds number. Difficulties with DGS are found for several problems. For driven cavity problems the multigrid method did not converge for $\mathrm{Re}>$ 100 [5]. Distributive $I L U$ smoothing on matrix $A B$ from (3.5) is performed in [35], with

$$
A B=L U-N,
$$


where $L U$ is an incomplete $L U$ factorization of $A B$. This smoother is robust and efficient. Another class of smoothing methods, not of distributive type, is introduced in [27]. The Symmetric Coupled Gauss-Seidel method (SCGS) is a method of collective Gauss-Seidel type. A system of coupled equations per cell $\left(2 * V^{1}\right.$-momentum, $2 * V^{2}$-momentum, and continuity) is updated cell by cell, as follows.

Suppose the residual $\mathbf{R}$ before a SCGS-update is given by

$$
\left(\begin{array}{l}
\mathbf{R}^{1} \\
\mathbf{R}^{2} \\
\mathbf{R}^{3}
\end{array}\right)=\left(\begin{array}{c}
\mathbf{F}^{1} \\
\mathbf{F}^{2} \\
0
\end{array}\right)-\left(\begin{array}{ccc}
A^{11} & A^{12} & A^{13} \\
A^{21} & A^{22} & A^{23} \\
A^{31} & A^{32} & 0
\end{array}\right)\left(\begin{array}{c}
\mathbf{V}^{1(n)} \\
\mathbf{V}^{2(n)} \\
\mathbf{p}^{(n)}
\end{array}\right)
$$

where $\left(\mathbf{V}^{1(n)}, \mathbf{V}^{2(n)}, \mathbf{p}^{(n)}\right)^{T}$ represents the current solution.

Corrections $\left[\delta \mathbf{V}^{1}, \delta \mathbf{V}^{2}, \delta \mathbf{p}\right]^{T}$ are calculated and added to the current solution. So:

$$
\left(\begin{array}{ccc}
A^{11} & A^{12} & A^{13} \\
A^{21} & A^{22} & A^{23} \\
A^{31} & A^{22} & 0
\end{array}\right)\left(\begin{array}{c}
\delta \mathbf{V}^{1} \\
\delta \mathbf{V}^{2} \\
\delta \mathbf{p}
\end{array}\right)=\left(\begin{array}{c}
\mathbf{R}^{1} \\
\mathbf{R}^{2} \\
\mathbf{R}^{3}
\end{array}\right)
$$

The system is solved with a Gauss-Seidel-type smoother per cell. For cell ( $i$, j):

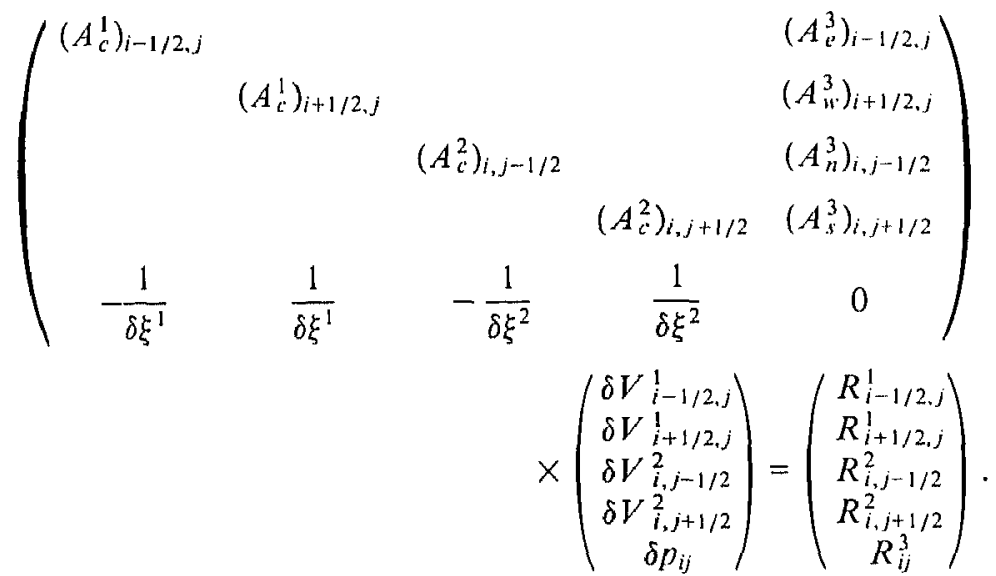

Underrelaxation is implemented as in [27]:

$$
\begin{gathered}
\left(A_{c}^{1}\right)_{i \pm 1 / 2, j}:=\left(A_{c}^{1}\right)_{i \pm 1 / 2, j} / \alpha_{1} \\
\left(A_{c}^{2}\right)_{i j \pm 1 / 2}:=\left(A_{c}^{2}\right)_{i j \pm 1 / 2} / \alpha_{2} .
\end{gathered}
$$


Equations (3.7) are solved with an explicit formula, after which the corrections are added to the current solution, the residual is updated, and the computation continues with the next cell.

The SCGS smoother performs very well for several problems in Cartesian domains [27-31, 36] and in arbitrarily shaped domains [12, 13]. However, it is a well-known fact that point Gauss-Seidel-type relaxation methods are not good smoothing methods in multigrid algorithms when the equations involve strong coupling of unknowns in some direction, which is the case when stretched cells occur. When the cell aspect ratio is high SCGS smoothing factors tend to one.

A line version of SCGS, in which only the pressures along a line are coupled is given in [20,23], and is called SCGS/LS (Line Solver). In arbitrarily shaped domains this is found not to be a good smoother when the aspect ratio is much larger than one. All unknowns in a line of cells must be updated simultaneously. An attempt by the authors of this article to construct a twostage smoother was not successful. In stage one the two momentum equations were updated with an Alternating Damped Line Jacobi method (ADLJ). This is a robust method with damping parameter 0.7 for the rotated convection diffusion equation and for the anisotropic diffusion equation [32]. In stage two the continuity was taken into account and the pressures were updated with SCGS or SCGS/LS. However, the underrelaxation parameter was problem dependent and large aspect ratios could still not be handled.

A fully coupled line version of SCGS is given in [26] and called Symmetric Coupled Alternating Lines (SCAL). All unknowns on a line of cells are updated simultaneously with alternating zebra-sweeping. The ordering is odd horizontal lines, even horizontal lines, odd vertical lines, even vertical lines. If for a sweep along horizontal lines the unknowns per line are ordered in the following way: $V_{i, j+1}^{2}, p_{i j}, V_{i, j}^{2}, V_{i+1, j}^{1}$, etc., the resulting matrix is a band matrix [26]. For the equations in curvilinear coordinates the band contains 13 elements (in Cartesian coordinates it is 9). In [26] it is reported that SCAL is much more robust than SCGS and less sensitive to variations of the underrelaxation parameters. This is the smoother implemented in our multigrid algorithm.

Underrelaxation is implemented in the "classical" way. Instead of calculating corrections $\delta \mathbf{V}$ new values $\mathbf{V}^{*}$ are being calculated and $\mathbf{V}^{(n+1)}$ is found as follows:

$$
\begin{aligned}
V^{1(n+1)} & =V^{1(n)}+\alpha_{1}\left(V^{1 *}-V^{1(n)}\right) \\
V^{2(n+1)} & =V^{2(n)}+\alpha_{2}\left(V^{2 *}-V^{2(n)}\right) \\
p^{(n+1)} & =p^{(n)}+\alpha_{3}\left(p^{*}-p^{(n)}\right) .
\end{aligned}
$$

The underrelaxation parameters $\alpha_{k}$ depend on the Reynolds number. 


\section{RESULTS}

Two types of flows have been investigated: driven cavity flows and channel flows. The discretization in curvilinear coordinates is tested on domains in which some grids with severe jumps in mesh size and mesh direction are put. For the multigrid solution algorithm average reduction factors $r_{n i t}$ are calculated, defined as

$$
r_{n i t}=\left(\frac{\left\|r e s_{n i t}\right\|}{\left\|r e s_{0}\right\|}\right)^{1 / n i t},
$$

i.e., the 2-norm of the residual after $n$ it iterations divided by the 2-norm of the starting residual to the power $(1 / n i t)$.

When the quantity $\left(\left\|r e s_{n}\right\| /\left\|r e s_{n-i}\right\|\right)$ becomes constant for $n \geqslant N$ (some iteration number) then this is the asymptotic convergence factor $\rho$, defined as

$$
\rho=r_{\infty} .
$$

The number of MG-iterations that were performed depended on the reduction factor $r_{n i t}$, as follows:

$$
\begin{aligned}
r_{n i t} \leqslant 0.15 ; & & n i t=10 \\
0.15<r_{n i t} \leqslant 0.25 ; & & n i t=15 \\
r_{n i t}>0.25 ; & & \text { nit }=20 .
\end{aligned}
$$

The multigrid schedule used in the W-cycle. For all problems the number of pre-smoothing iterations is 1 , as is the number of post-smoothing iterations. On the coarsest grid the problem is solved "exactly" by performing many smoothing iterations ( 10 or more). Underrelaxation parameters depend only on the Reynolds number. For $\mathrm{Re} \leqslant 150, \alpha_{k}=0.7$ for $k=1,2,3$ seems to be good and gives level-independent reduction factors. For $\mathrm{Re}=1000, \alpha_{k}=0.4$ for $k=1,2$ and $\alpha_{3}=1.0$.

\section{Driven Cavity Problems}

First the driven cavity flow in a square cavity is investigated. To study the robustness of the smoother the grids were strongly refined near the boundaries. Four grids were tested:

(i) the equidistant grid;

(ii) a grid in which the boundary cells have aspect ratio 10, see Fig. 4.1; 


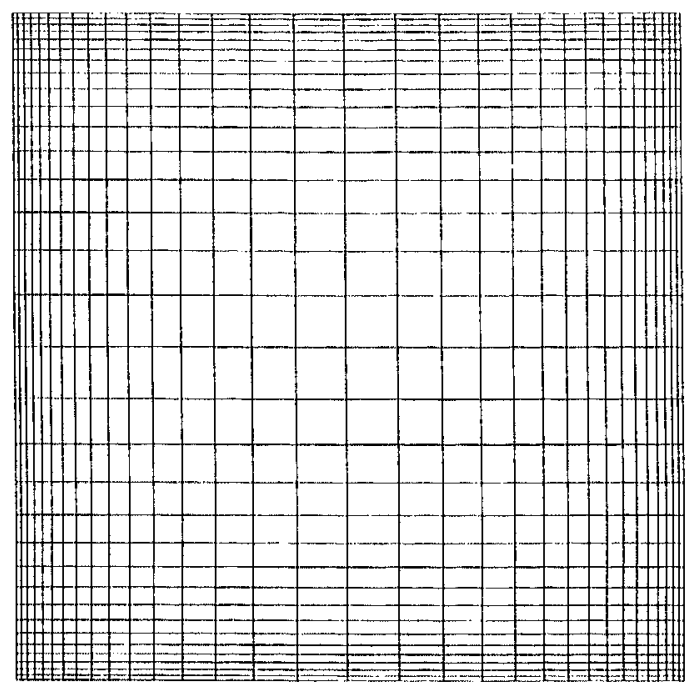

FIG. 4.1. The $32 \times 32$ mesh with aspect ratio 10 at the boundary.

(iii) the boundary cells have aspect ratio 100 ;

(iv) the boundary cells have aspect ratio 1000 .

This is a severe test on robustness; stretched cells occur in all directions.

Table 4.1 shows $r_{n i t}$ and (where available) $\rho$ for grids with 5 to 7 levels. The average reduction factors are to a satisfactory extent level independent and for many aspect ratios and many Reynolds numbers below .25. The performance of SCGS (not shown) is poor in the cases with large aspect ratios.

In Table 4.2 the CPU times on a Convex 3820 computer are presented for one smoothing iteration and one W-cycle. Parallelization is not employed here, further no special ordering strategies for the data structure are employed to profit from vectorization. From Table 4.2 it can be seen that the work for a W-cycle is about 4.8 times the work for one smoothing iteration, which is not surprising, since the theoretical ratio counting only smoothing work would be 4 in this case.

Another interesting cavity problem is the flow through a lid driven L-shaped cavity. This cavity problem causes extra problems, because boundary II and boundary IV, see Fig. 4.2, consist of two parts. The top half of $\Gamma_{2}$ is moving from right to left. This problem is suited for a multidomain solution technique application, which is shown in [16]. For a single domain discretization in curvilinear coordinates it is a severe test case due to the $90^{\circ}$ change in mesh direction. The grid is shown in Fig. 4.2. The grid has been made non-smooth on purpose. Table 4.3 shows $r_{n i t}$ for two fine meshsizes for a flow at $\operatorname{Re}=100$ and $\operatorname{Re}=1000$. Figure 4.3 shows the streamlines for both cases. 
TABLE 4.1

Average and Asymptotic Reduction Factors for Driven Cavity Problems

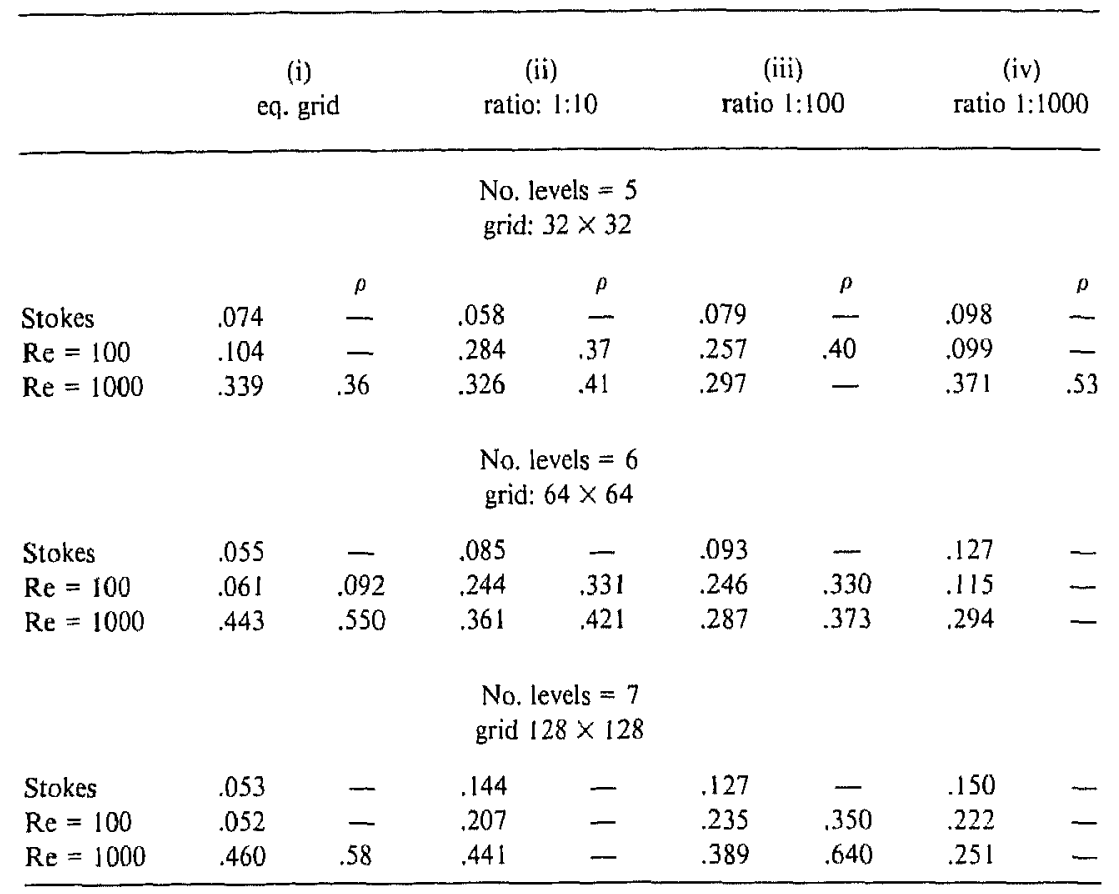

The reduction factors of the SCAL smoother are much better than for SCGS [12], while the cost of using the line solver is only about $50 \%$ larger than the SCGS solver [26]. Furthermore, SCAL is more robust in the choice of domain parameters. For this complicated flow problem $r_{n i}$ is good and the discretization seems accurate.

\section{Channel Flow Problems}

Because only Dirichlet boundary values are implemented in the code at present we are somewhat restricted. However we are still able to investigate

TABLE 4.2

CPU TIMES ONA CONVEX 3820

\begin{tabular}{lccc}
\hline Cycle grid & $32 \times 32$ & $64 \times 64$ & $128 \times 128$ \\
\hline $1 \mathrm{sm}$. & $0.33 \mathrm{~s}$ & $1.24 \mathrm{~s}$ & $4.40 \mathrm{~s}$ \\
$\mathrm{~W}$ & $1.56 \mathrm{~s}$ & $5.47 \mathrm{~s}$ & $21.4 \mathrm{~s}$ \\
\hline
\end{tabular}




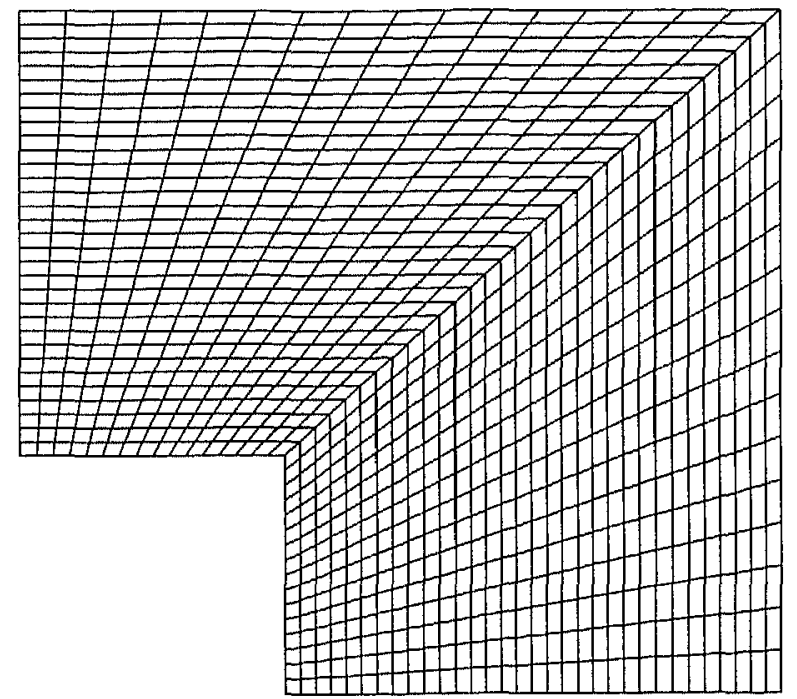

FIG . 4.2. The mesh for an L-shaped cavily.

some interesting channel flow problems. For all channel flow problems nested iteration [6] is used, so the algorithm starts on the coarsest grid. First the flow through a straight channel is investigated. Results for this simple case are presented only for comparison with more complicated cases. Figure 4.4 shows a sketch of the channel. The length of the channel $L=100$; the width $d=1$. An equidistant grid with cells of aspect ratio 100 is put over the domain. Parabolic in- and outflow velocity profiles are prescribed. The exact Poisseuille flow solution is recovered accurately. The pressure distribution is linear, as expected.

Table 4.4 presents $r_{n i t}$ for the Stokes flow and for a flow at $\operatorname{Re}=1000$. These reduction factors are satisfactory and level independent, whereas SCGS reduction factors are close to $1(r=0.992)$.

\section{Flow over a Cylindrical Protuberance}

Another problem is the linear shear flow over a cylindrical protuberance in a channel (Fig. 4.5). This flow is a two-dimensional version of the flow over a spherical protuberance in the viscous sublayer in a turbulent boundary layer, which is of interest to turbulent flow researchers. The problem is artificial with only Dirichlet boundary conditions. We are restricted to a Stokes flow. However, it is interesting to show the possibilities of a curvilinear discretization for this case. Again a multidomain approach is a natural approach but we forego this approach on purpose here, to study multigrid convergence on a contorted grid. Figure 4.6 shows the $16 \times 40$ mesh. Boundary $\Gamma_{4}$ consists of 

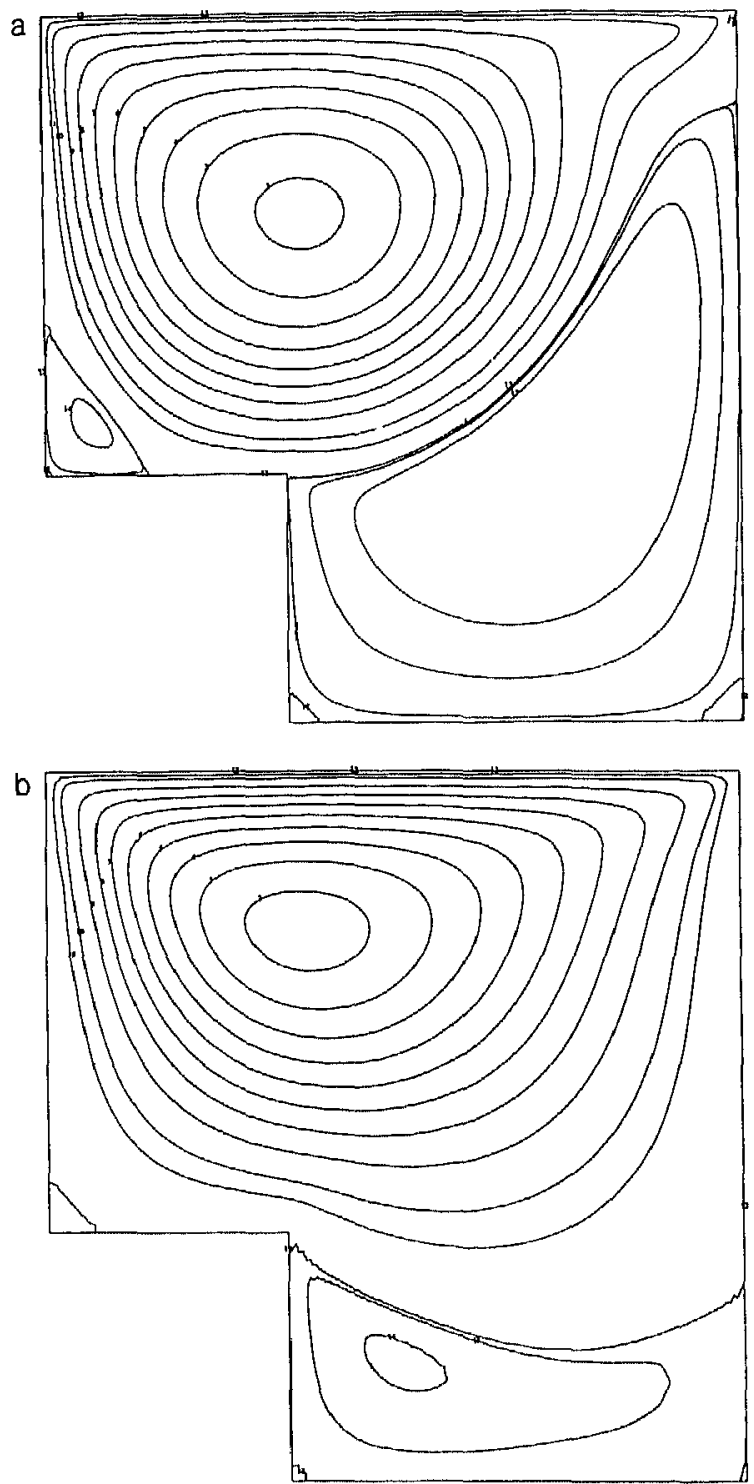

FIG . 4.3. Streamlines for the L-shaped cavity flow at (a) $\operatorname{Re}=100$ and (b) $R e=1000$.

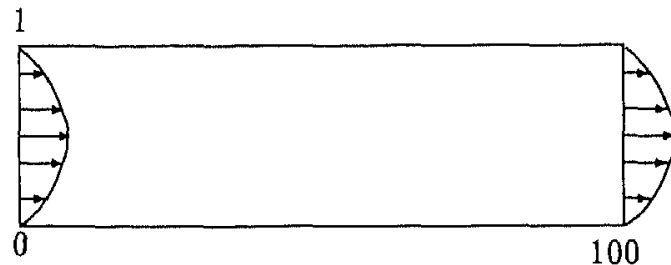

FIG. 4.4. Sketch of the domain for the straight channel. 
TABLE 4.3

AVERAGE REDUCTION FACTORS For a Flow IN AN L-SHaped CAVITY

\begin{tabular}{cccc}
\hline Levels & Grid & $\operatorname{Re}=100$ & $\operatorname{Re}=1000$ \\
\hline 6 & $64 \times 64$ & .21 & .48 \\
7 & $128 \times 128$ & .19 & .52 \\
\hline
\end{tabular}

three parts, where on the in- and outflow part a linear velocity profile is prescribed:

$$
u_{n}=y ; \quad u_{t}=0
$$

On the upper boundary $u_{l}$ is prescribed: $u_{t}=y ; u_{n}=0$, while on boundary $\Gamma_{1}, \Gamma_{2}$, and $\Gamma_{3} u_{n}=u_{t}=0$. The multigrid algorithm performs well, and gives $r_{10}=0.105$. Figure 4.7. shows streamlines and isobars for this problem.

\section{The Hole-Pressure Problem}

A rather difficult channel flow problem is the so-called hole-pressure problem [9]. The flow domain is depicted in Fig. 4.8. It consists of a uniform channel of height $d$ and length $L$, into which a cavity of width $b$ and depth $h$ has been cut. Dirichlet boundary conditions are prescribed on the whole boundary. In [9] this hole-pressure problem is proposed as new test case for numerical codes. The problem is also of practical importance: It is the basis of a scientific instrument to help characterizing non-Newtonian fluids.

The mathematical and experimental problem is to determine the difference between the normal stress acting on the bottom of the slot and that acting on the upper wall above the opening to the slot. This quantity is the so-called "hole pressure" $\Delta$, given by

$$
\Delta=\frac{1}{s_{1}} \int_{s_{1}}-p(x, 1) d x-\frac{1}{s_{2}} \int_{s_{2}}-p(x,-d) d x .
$$

TABLE 4.4

average Reduction Factors for a Flow Through a Straight Channel. WITH A MESH WITH ASPECT RATIO 100

\begin{tabular}{ccc}
\hline Grid & $r$, Slokes & $r, \operatorname{Re}=1000$ \\
\hline $32 \times 32$ & .122 & .463 \\
$64 \times 64$ & .126 & .487 \\
$128 \times 128$ & .129 & .500 \\
\hline
\end{tabular}




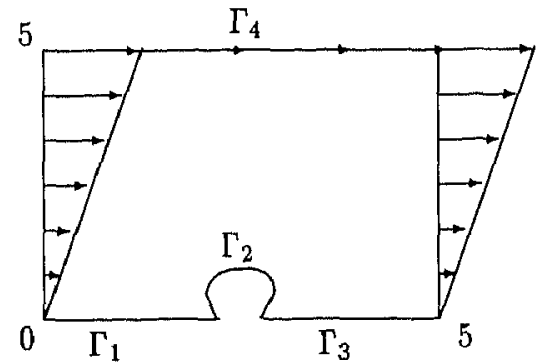

FIG. 4.5. A sketch of the problem of a flow over a cylindrical protubcrance.

The geometry of the reference domain is defined by: $L=5 ; d=1 ; b=1 ; h$ $=1 ; s_{1}=s_{2}=1$. The boundary conditions are given by:

$$
\begin{array}{ll}
\text { On } \Gamma_{2} \text { and } \Gamma_{4}: & u_{n}=0 ; u_{t}=0 \\
\text { On } \Gamma_{1} \text { and } \Gamma_{3}: & u_{n}=y(1-y) ; u_{t}=0 .
\end{array}
$$

So $U^{\max }=\frac{1}{4} ; U^{\text {average }}=\frac{1}{6}$ and the mass flow $Q=\frac{1}{6}$. For the Reynolds number, defined as $\operatorname{Re}=Q / \nu$, it follows that $\operatorname{Re}=1 / 6 \nu$.

The mesh is generated as follows. Boundary part $\Gamma_{4}$ is divided into five parts (see Fig. 4.9). Each part is connected to a part of $\Gamma_{2}$ in the manner shown in the figure. This results in a coarse mesh, which is refined to a $32 \times$ 112 mesh, which is then smoothed a little. Figure 4.10 shows a part of this mesh. In [9] hole pressures are presented up to $R e=10$ calculated with a finite element code on a very fine mesh. However the hole pressures from [9] are not calculated with the boundary conditions from [9], but with the boundary conditions presented here [17]. In Table 4.5 the calculated hole pressures from the $32 \times 112$ mesh are compared to them. Figure 4.11 presents

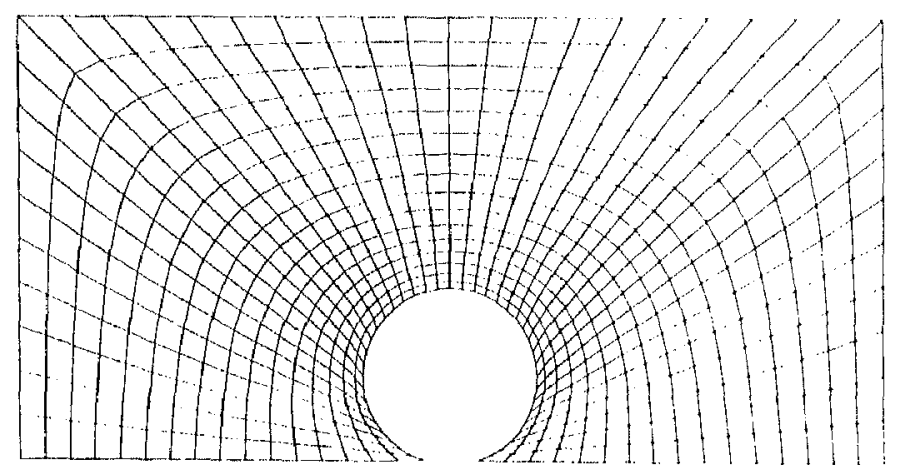

FIG. 4,6. The mesh for a flow over a cylindrical protuberance. 
MULTIGRID FOR NAVIER-STOKES EQUATIONS
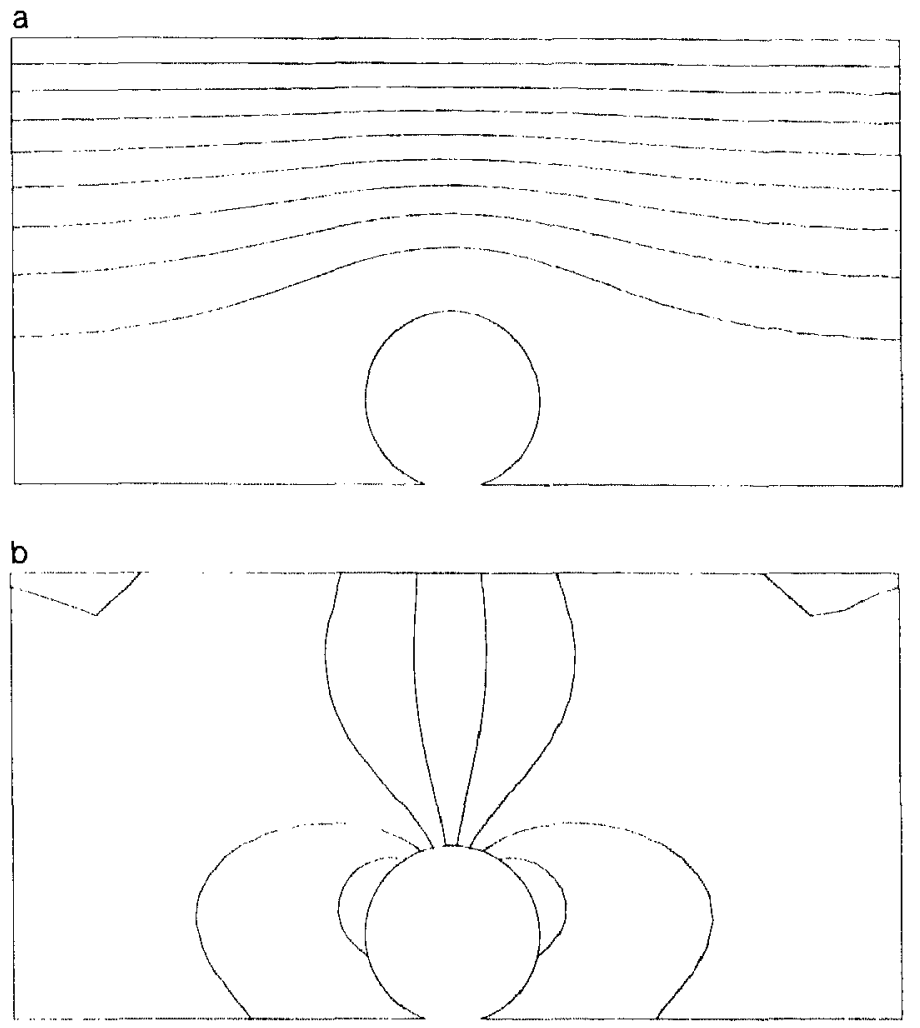

FIG. 4.7. Streamlines and isobars for a Stokes flow over a cylindrical protuberance.

streamlines and the isobars for $\operatorname{Re}=10$. They resemble the distributions in [9] very well. The multigrid performance is satisfactory as well: $r_{15}=0.247$ for $\operatorname{Re}=10$ and $r_{25}=.505$ for $\operatorname{Re}=100$.

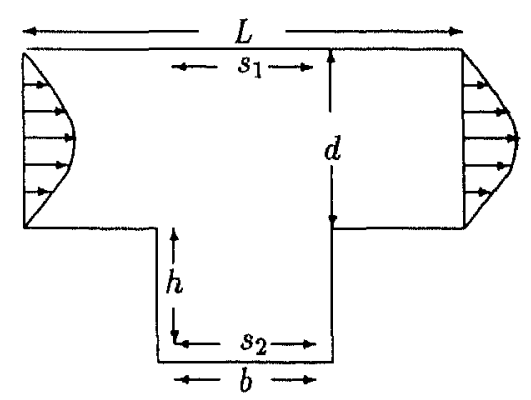

FiG. 4.8. The domain for the hole-pressure problem. 


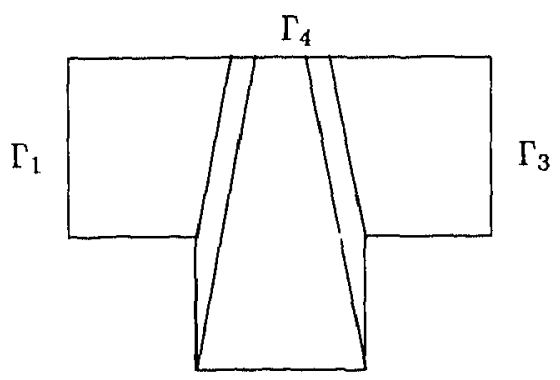

$\Gamma_{2}$

FIG. 4.9. A sketch of the parts of the domain, which are connected when the mesh is generated.

\section{Backward-Facing Step}

Another flow, heavily studied over the years, is laminar flow over a backward-facing step. A GAMM workshop was held in 1983 in Bièvres on this flow problem [10]. Four cases were studied there, two of them are examined here in curvilinear coordinates, namely, cases 2 and 4 as defined in [10]. The domain for these two cases is sketched in Fig. 4.12.

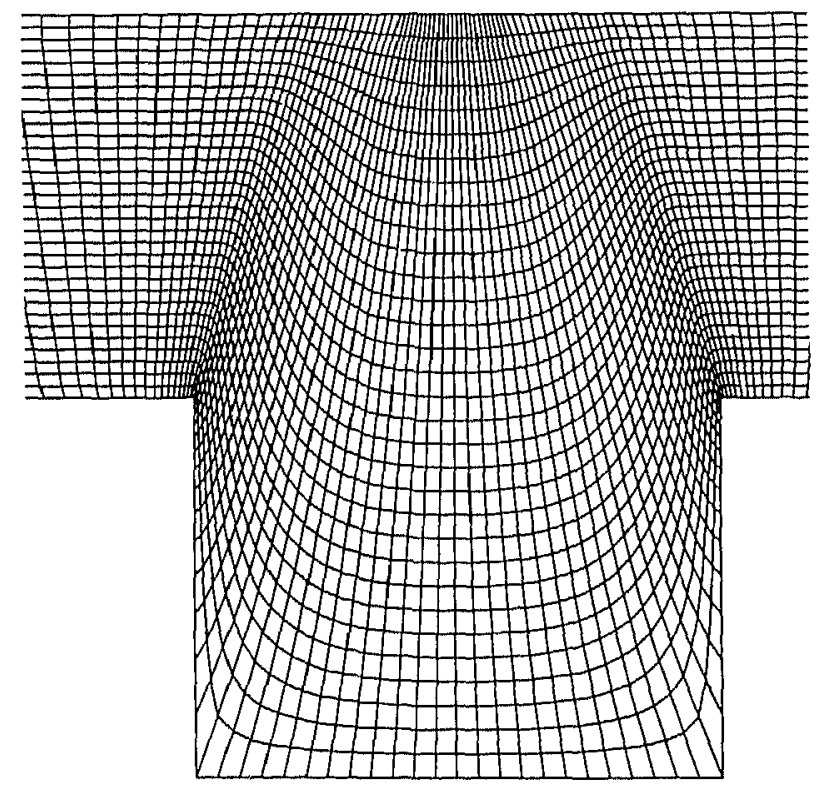

FIG, 4.10. A part of the $32 \times 112$ mesh for the hole-pressure problem. 
TABLE 4.5

Hole Pressure $\Delta$ Compared with [9] for SEVERal Reynolds Numbers

\begin{tabular}{rcc}
\hline $\operatorname{Re}$ & $\Delta$ calc. & $\Delta[9]$ \\
\hline 1 & 0.064 & 0.049 \\
5 & 0.236 & 0.226 \\
10 & 0.426 & 0.383 \\
\hline
\end{tabular}

a

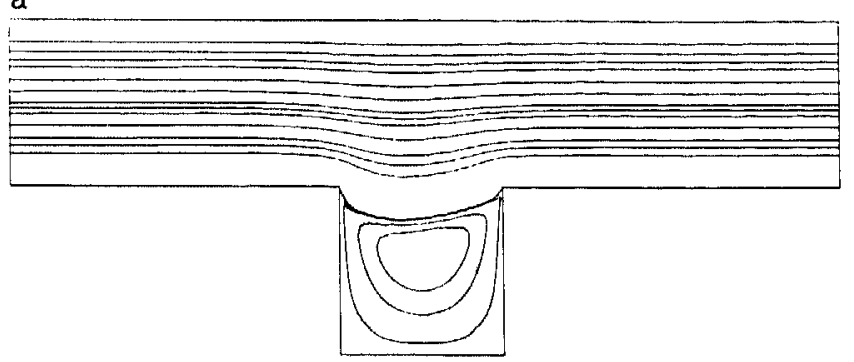

b

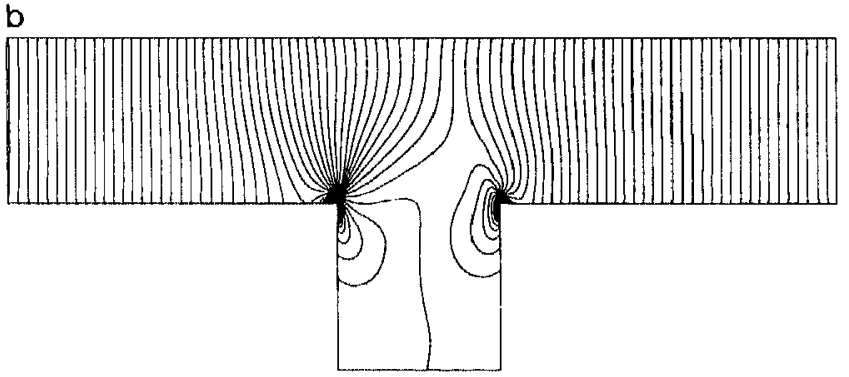

FIG. 4.11. Streamlines and isobars for the hole-pressure problem.

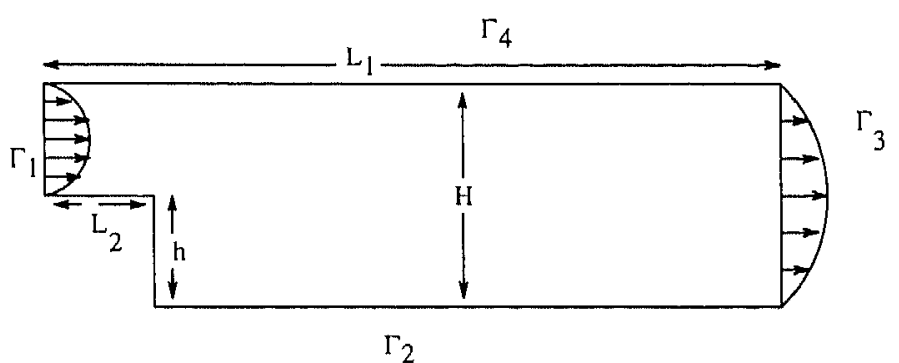

FIG. 4.12. The domain (not to scale) for the flow over a backward facing step. 


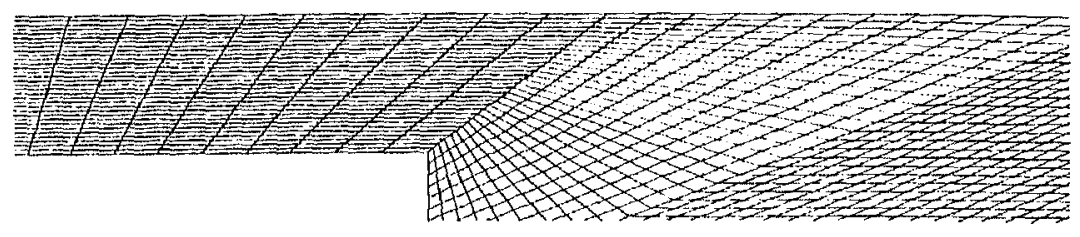

FIG. 4.13. A part of the $32 \times 64$ mesh.

The geometrical parameters are: $L_{1}=22, L_{2}=3, H=1$ and $h=0.5$. Computations presented are for $\operatorname{Re}=50$ and $\operatorname{Re}=150$, with the Reynolds number defined as

$$
\operatorname{Re}=U^{\max } \cdot(H-h) / \nu,
$$

with $U^{\max }$ the maximum value of the velocity at the entrance, (the velocity profile is prescribed: $u_{n}=16 y(0.5-y)$ at the entrance and $u_{n}=2 y(1-y)$ at the outlet; $\left.u_{t}=0\right)$ and $\nu(=\mu / \rho)$ the viscosity.

Figure 4.13 shows a part of the $32 \times 64$ mesh. Deliberately, no attempt was made to make this mesh more smooth. In Fig. 4.14 the isobars and streamlines for the flow at $\mathrm{Re}=50$ are given, together with a more detailed picture of the streamlines in the recirculation zone. In Fig. 4.15 these results are shown for the flow at $\mathrm{Re}=150$. In Table 4.6 the MG performance is

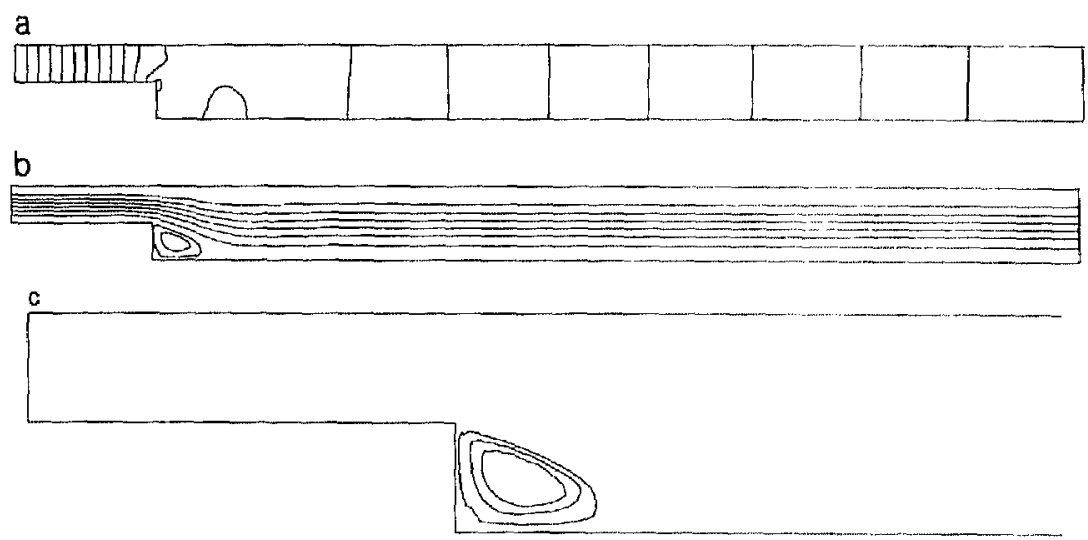

FlG. 4.14. Isobars and streamlines for $\mathrm{Re}=50$. 


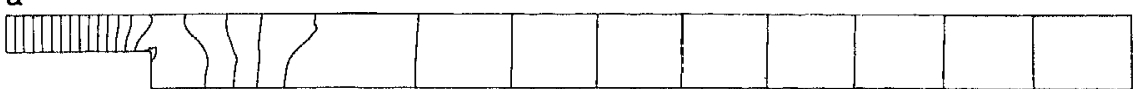

b
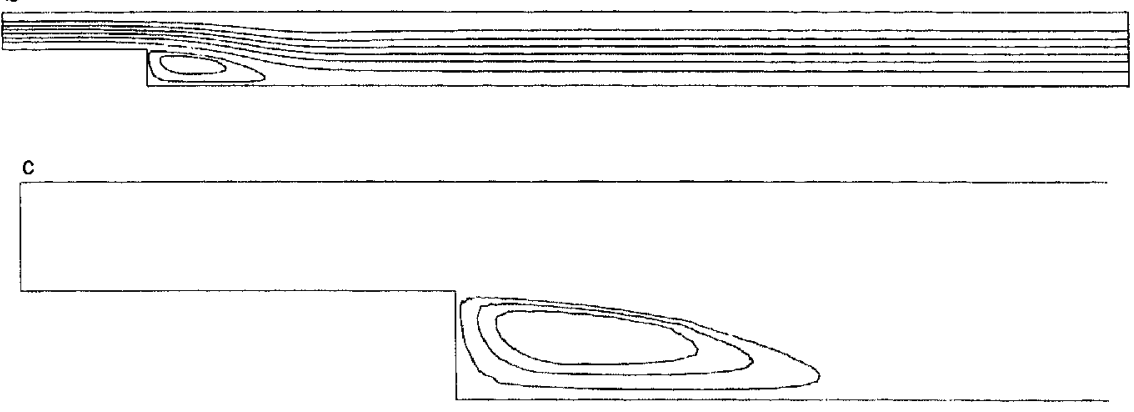

FIG , 4.15. Isobars and streamlines for $\mathrm{Re}=150$.

given for a $16 \times 32$ grid and a $32 \times 64$ grid. The coarsest grid is taken to be the $4 \times 8$ grid. For the $32 \times 64$ grid the length of the recirculation zone, scaled by $(H-h)$ is calculated and presented in Table 4.6. This length resembles the length found by many of the GAMM contributors very well [10]. The reduction factors look satisfactory and level independent.

\section{CONCLUSIONS}

The discretization of the incompressible Navier-Stokes equations in curvilinear coordinates shows accurate results for many geometries and fairly nonuniform grids. A robust smoother is constructed, which can deal with cells of varying size coming from a mesh generator. Multigrid reduction factors for flows in domains in which stretched cells occur are good, well below 1,

TABLE 4.6

Avirage Reduction Factors and REattachment Point for a Flow over a BACKWARD-FACING STEP

\begin{tabular}{lccc}
\hline & $r_{20}(16 \times 32)$ & $r_{20}(32 \times 64)$ & Reattachment point \\
\hline $\operatorname{Re}=50$ & .449 & .482 & 2.03 \\
$\operatorname{Re}=150$ & .654 & .656 & 5.00 \\
\hline
\end{tabular}


contrary to the reduction factors of SCGS in these domains. The SCAL smoother is more robust in the variation of underrelaxation factors than the SCGS smoother.

\section{REFERENCES}

1. R. Aris, Vectors, Tensors and the Basic Equations of Fhid Mechanics. Prentice-Hall, Englewaod Cliffs, NJ (1962).

2. A. Brandt, Guide to multigrid development. In W. Hackbusch and U. Trottenberg (Eds.), Muthigrid Methods, Ser. Lecture Notes in Mathematics, Vol. 960, pp. 220-312. Springer, Berlin (1982).

3. A. Brandt and N. Dinar, Multigrid solutions to flow problems. In S. Parter (Ed.), Numerical Methods for Partial Differential Equations, pp. 53-147. Academic Press, New York (1979).

4. L. Davidson and P. Hedberg, Mathematical derivation of a finite volume formulation for laminar flow in complex geometries. Internat. J. Numer. Methods Fluids 9, 531-540 (1989).

5. L. Fuchs, Multi-grid schemes for incompressible flows. In W. Hackbusch (Ed.), Efficient Solutions of Ellipic Systems, Ser. Notes on Numerical Fluid Mechanics, Vol. 10, pp. 38-51. Vieweg, Braunschweig (1984).

6. W. Hackbusch, Multi-grid Methods and Applications. Springer-Verlag, Berlin (1985).

7. K. Katsuragi and O. Ukai, Navier-Stokes equations on a curvilinear coordinate system. $\mathrm{ln}$ P. Wesseling (Ed.), Proc. of the 8th GAMM Conf. on Num. Meth. in Fluid Mech. Ser. Notes on Num. Fluid Mech., Vol. 29. Vieweg, Braunschweig (1990).

8. S. Koshizuka, Y. Oka, and S. Kondo, A staggered differencing technique on boundary-fitted curvilinear grids for incompressible flows along curvilinear or slant walls. Comput. Mech. 7, 123-136(1990).

9. A. S. Lodge, W. G. Pritchard, and L. R. Scout, The hole-pressure problem. IMA J. Appl. Math. 46, 39-66 (1991).

10. K. J. Morgan, J. Periaux, and F. Thomasset (Ed.), Analysis of Laminar Flow over a Backward Facing Slep, GAMM Workshop held at Bievres (Fr.), Vieweg, Braunschweig (1984).

11. A. E. Mynett, P. Wesseling, A. Segal, and C. G. M. Kassels, The ISNaS incompressible Navier-Stokes solver: invariant discretization. Appl. Sci. Res. 48, 175-191 (1991).

12. C. W. Oosterlee and $P$. Wesseling, A multigrid method for a discretization of the incompressible Navier-Stokes equations in general coordinates. In J. B. Vos, A. Rizzi, I. L. Rhyming (Eds.), Proc. of the 9th GAMM Conf. on Num. Meth. in Fluid Mech. Ser. Notes on Num. Fluid Mech., Vol. 35, pp. 99-106. Vieweg, Braunschweig (1992).

13. C. W. Oosterlec and P. Wesseling, A Muligrid Method for an Invariant Formulation of the Incompressible Navier-Slokes Equations in General Coordinates. Comm. Appl. Numer. Melhods 8, 721-724 (1992).

14. S. V. Patankar and D. B. Spalding, A calculation procedure for heat and mass transfer in three-dimensional parabolic flows. Internat. J. Heat Mass Transfer 15, 1787-1806 (1972).

15. S. V. Patankar, Numerical Heat Transfer and Fluid Flow. McGraw-Hill, New York (1980).

16. C. Y. Perng and R. L. Street, A coupled multigrid-domain-splitting technique for simulating incompressible flows in geometrically complex domains. Internat. J. Numer. Methods Fltids $13,269-286$ ( 1991 ).

17. W. G. Pritchard, Private communication. 
18. D. Rayner, Multigrid flow solutions in complex two-dimensional geometries. Internat. $J$. Numer. Methods Fluids 13, 507-518 (1991).

19. W. Rodi, S. Majumdar, and B. Schönung, Finite volume methods for two-dimensional incompressible flows with complex boundaries. Comput. Methods Appl. Mech. Engrg. 75, 369392 (1989).

20. J. I. Rollet, D. F. Mayers, and T. M. Shah, Analysis and Application of an Efficient Line Solver Based upon the Symmetric Coupled Gauss-Seidel Scheme. Report 88/10, Oxford Univ. Comp. Lab. (1988).

21. M. Rosenfeld, D. Kwak, and M. Vinokur, A solution method for the unsteady incompressible Navier-Stokes equations in generalized coordinate systems. J. Compul. Phys. 94, 102-137 (1991).

22. A. Segal, P. Wesseling, J. Van Kan, C. W. Oosterlee, and K. Kassels, Invariant discretization of the incompressible Navier-Stokes equations in boundary fitted co-ordinates. Internat. $J$. Numer. Methods. Fluids 15, 411-426 (1992).

23. T. M Shah, Analysis of a Multigrid Method. Master's thesis, Oxford Univ. Comp. Lab. (1987).

24. G. J. Shaw and S. Sivaloganathan, On the smoothing of the SIMPLE pressure correction algorithm. Internat. J. Numer. Methods Fluids 8, 441-462 ( [988).

25. I. S. Sokolnikoff, Tensor Analysis. Wiley, Englewood Cliffs, NJ (1964).

26. M. C. Thompson and J. H. Ferziger, An adaptive multigrid technique for the incompressible Navier-Stokes equations. J. Comput. Phys. 82, 94-121 (1989).

27. S. P. Vanka, Block implicit multigrid calculation of two-dimensional recirculating flows. Comput. Methods Appl. Mech. Engrg. 59, 29-48 (1986).

28. S. P. Vanka, Block-implicit calculation of steady turbulent recirculating flows. Internat. $J$. Heat Mass Trans/er 28, 2093-2103 (1985).

29. S. P. Vanka, Block-implicit multigrid solution of Navier-Stokes equations in primitive variables. J. Comput. Phys. 65, 138-158(1986).

30. S. P. Vanka, A calculation procedure for three-dimensional steady recirculating flows using multigrid methods. Comput. Methods Appl. Mech. Engrg. 59, 321-338 (1986).

31. S. P. Vanka and K. Misegades, Vectorized Mulligrid Fluid Flow Calculations on a CRAYXMP48. AlAA Paper 86-0059 (1986).

32. P. Wesseling, An Introdtution to Multigrid Methods. Wiley, Chichester (1992).

33. P. Wesseling, A. Segal, J. V. Kan, C. Oosterlee, and C. Kassels. Finite volume discretization of the incompressible Navier-Stokes equations in general coordinates on staggered grids. Comput. Fhids Dynamics J. 1, 27-33 (1992).

34. G. Wittum, Mchrgitterverfahren für die Stokessche Gleichung. Z. angew. Math. Mech. 67. 499-50I (1987).

35. G. Wittum, Multi-grid methods for Stokes and Navier-Stokes equations with transforming smoothers: Algorithms and numerical results. Numer. Math. 54, 543-563(1989).

36. N. Wright, Multigrid Solutions of Elliptic Fluid Flow Problems. Ph.D. thesis, Leeds University (1988). 